\title{
FECUNDIDADE DAS MULHERES CABO-VERDIANAS - ÁFRICA OCIDENTAL+
}

\section{CAPE VERDEAN WOMEN'S FERTILITY - WEST AFRICA}

Carlos Mendes Tavares*

Ana Amelia Camarano**

Luiz Carlos de Abreu***

Tavares MC, Camarano AM, Abreu LCde. Fecundidade das mulheres cabo-verdianas - África Ocidental. Rev Bras Crescimento Desenvol Hum. 2008; 18(1): 01-10.

\begin{abstract}
Resumo: Os níveis de fecundidade de um país fazem parte de um elenco de indicadores que orientam na formulação de políticas públicas, face à redução do volume da população e do seu envelhecimento. $\mathrm{O}$ aumento da fecundidade pode indicar falta de acesso da população a informações e serviços de saúde reprodutiva. Assim, o objetivo é analisar a fecundidade das mulheres cabo-verdianas e a contribuição de determinantes próximos da fecundidade. Realizouse a análise e a mensuração do comportamento da fecundidade das mulheres a partir de métodos indiretos de estimação da fecundidade com base nos dados dos censos. A análise dos determinantes próximos da fecundidade é baseado no DHS (Pesquisa sobre Saúde e Demografia - 1998). Os dados mostraram uma redução de 1,7, 1,5 e 1,6 filhos por mulher no final do período reprodutivo, de acordo com os três métodos (Brass, Arriaga e Gompertz) entre 1990 e 2000. As taxas foram mais altas para as mulheres das áreas rurais. A fecundidade vem diminuindo no país e com ocorrência mais precoce. O uso de contraceptivos foi o determinante de maior relevância no processo de declínio de fecundidade das mulheres de Cabo Verde, África.
\end{abstract}

Palavras-chave: Taxa de fecundidade; anticoncepção; fertilidade; Africa Ocidental.

\section{INTRODUÇÃO}

A fecundidade é uma das duas variáveis demográficas determinantes do crescimento vegetativo de uma dada população. Estudos sobre níveis e padrões de fecundidade são extremamente importantes, uma vez que mostram a situação e as tendências em relação ao número de filhos ti- dos e indicam a distribuição relativa dos nascimentos entre as mulheres em idade reprodutiva. Além disso, os padrões de fecundidade que se estabelecem a partir do nascimento do primeiro filho e do tamanho final da família são, em grande parte, responsáveis pela probabilidade de sobrevivência dos filhos, pela morbimortalidade nos primeiros anos de vida e pela saúde da mãe. ${ }^{1}$

\footnotetext{
Mestre em Estudos Populacionais e Pesquisas Sociais. Instituto Nacional de Estatística de Cabo Verde, África Ocidental.

** Demógrafa. Pós-Doutorado em Demografia. Professora do programa de Pós-graduação stricto sensu da Escola Nacional de Ciências Estatísticas. Técnica em pesquisa e planejamento do Instituto de Pesquisa Econômica Aplicada do Distrito Federal, Brasil.

*** Pós-Doutorado em Saúde Pública. Professor do Departamento de Fisiologia da Faculdade de Medicina do ABC, Santo André, SP., Brasil.

Correspondência: Carlos Mendes Tavares. Instituto Nacional de Estatística - INE/República de Cabo Verde, África Ocidental. Avenida Amilcar Cabral, S/N. Caixa Postal 116. Cidade da Praia - Ilha de Santiago, Cabo Verde - África Ocidental.e-mail: tavares.is.c@gmail.com

+ Parte dos resultados da dissertação, em nível de mestrado, do Programa de Pós-Graduação da Escola Nacional de Ciências Estatística do Instituto Brasileiro de Geografia e Estatística, Rio de Janeiro, Brasil. Defendida em março de 2005.
} 
As informações referentes aos níveis e aos padrões de fecundidade são indispensáveis para o estudo das perspectivas de crescimento da população de um país, assim como para a avaliação da sua estrutura por idade e variações, ${ }^{2}$ além de fornecer subsídios para análises que investigam o comportamento reprodutivo de uma população, que são, por conseguinte, fundamentais para a elaboração e para a implantação de políticas públicas voltadas para a saúde materna e infantil. $^{3}$

Nesse contexto, há que sublinhar a relevância de se concentrar análises desse fenômeno em torno de grandes linhas, tais como evolução da fecundidade total, por idade e seus diferenciais, além do estudo dos determinantes próximos da fecundidade. Assim, o objetivo deste estudo é analisar a fecundidade das mulheres cabo-verdianas e descrever a contribuição de determinantes próximos da fecundidade nessa população.

\section{MÉTODO}

Cabo Verde é um país africano, arquipélago de origem vulcânica, constituído por dez ilhas. Está localizado no Oceano Atlântico. A população residente, segundo estimativas do Instituto Nacional de Estatística de Cabo Verde (INE), é cerca de 480.000 habitantes em 2006 em uma proporção de $48 \%$ homens e $52 \%$ mulheres.

Considerando a ausência das estatísticas de nascimento de qualidade em Cabo Verde, por conta da baixa cobertura do registro civil para a mensuração dos níveis e tendências da fecundidade, optou-se por utilizar os dados dos censos de 1990 e 2000. Esses dados são referentes: 1) ao número de mulheres por grupo qüinqüenal; 2 ) ao número de filhos tidos nos últimos 12 meses que antecedem a data da realização do censo; e 3) ao número de filhos nascidos vivos, que constituem fonte para o cálculo de medidas indiretas de fecundidade.

Em Cabo Verde, assim como na maioria dos países africanos e subdesenvolvidos, há impossibilidade de obtenção desses indicadores por meio de estatísticas correntes de nascimentos, face aos problemas inerentes à existência de registros tardios e de sub-registros de nascimentos, o que impõe aos estudos sobre fecundidade valerem-se de quesitos especiais levantados em censos demográficos ou pesquisas, como fontes alternativas para a obtenção de tais indicadores. Entre as técnicas indiretas conhecidas para estimar os níveis de fecundidade, foram escolhidas as propostas por Brass, ${ }^{4}$ Arriaga ${ }^{5}$ e Gompertz. ${ }^{6}$

\section{Método de fecundidade proposto por Brass ${ }^{4}$}

Albuquerque et al., ${ }^{7}$ afirma que:

Esse método fornece a estimativa da fecundidade do grupo de mulheres que estão expostas ao risco de ter filhos, aceitando-se o padrão etário das taxas específicas de fecundidade $f_{i}, \mathrm{i}=1,2, \ldots, 7$, as quais são derivadas da razão entre o número de filhos tidos nos últimos 12 meses que antecedem a data de referência do inquérito e o número de mulheres classificadas por grupos qüinqüenais de idade. Por outro lado, o nível de fecundidade é estimado por meio do número médio de filhos tidos nascidos vivos informado pelas mulheres mais jovens. ${ }^{\text {? }}$

A aplicação dessa técnica, entretanto, depende de duas condições básicas: a constância de fecundidade e a de mortalidade. Além dessas, ela assume:

§ A imprecisão no período de referência, a principal fonte de erro em relação às informações sobre nascimentos ocorridos no ano anterior;

$\S$ As mulheres mais jovens, as quais tendem a informar com maior precisão o número de filhos nascidos vivos. Há uma marcante evidência de omissão de uma proporção de filhos tidos pelas mulheres informantes na medida em que estas vão envelhecendo.

\section{Método de fecundidade proposto por Arri- aga $^{5}$}

Arriaga $^{5}$ propõe uma técnica alternativa para estimar a fecundidade em caso da existência de informações de dois levantamentos consecutivos sobre número de filhos tidos nascidos vivos, classificados segundo a idade da mãe. Ao contrário da técnica de $\mathrm{Brass}^{4}$, que supõe fe- 
cundidade constante, a hipótese implícita na técnica proposta por Arriaga ${ }^{5}$ é que o número médio de filhos nascidos vivos por mulher varia linearmente no intervalo de tempo considerado.

\section{Método de fecundidade proposto por Gom- pertz $^{6}$}

De acordo com Newell, ${ }^{6}$ o sistema de Gompertz relacionado à fecundidade é uma técnica de estimação indireta das taxas de fecundidade. Esse método apresenta similaridades ao sistema do lógico relacional de tábua de vida, em que é expressa uma transformação linear para se obter um padrão estimado, por meio de dois parâmetros, a2. Os modelos relacionais expressam a fecundidade em função unicamente da idade.

O modelo de Bongaarts ${ }^{8}$, mais comumente utilizado, foi aplicado para cálculo e análise dos determinantes próximos da fecundidade. Bongaarts $^{8}$ (1978) relaciona sete determinantes: i) a proporção de mulheres casadas ou unidas; ii) a prevalência do uso de anticoncepção; iii) abortos provocados; iv) infertilidade pós-parto; v) intervalo de tempo para a concepção; vi) risco de mortalidade intra-uterina; e vii) o início da esterilidade permanente. ${ }^{8,9}$

\section{RESULTADOS}

Nas tabelas 1, 2 e 3, as taxas de fecundidade total estimadas para Cabo Verde, que em meados de 1990 eram de 5,8, 5,5 e 5,7 filhos, pelos métodos de Brass, ${ }^{4}$ Arriaga ${ }^{5}$ e Gompertz, ${ }^{6}$ respectivamente, declinaram para 4,1 filhos, pelo primeiro e pelo terceiro métodos, e 4,0, para o segundo, em 2000. Esses dados mostram uma redução de 1,7, 1,5 e 1,6 filhos na taxa de fecundidade entre 1990 e 2000 , de acordo com as técnicas de Brass, ${ }^{4}$ Arriaga ${ }^{5}$ e Gompertz,${ }^{6}$ respectivamente.

As taxas de fecundidade total para as mulheres urbanas em 1990 eram de 5,2 filhos, enquanto para as rurais eram de 4,8 filhos. Em 2000, medidas pelos métodos de $\mathrm{Brass}^{4}$ e Arria$\mathrm{ga}^{5}{ }^{5}$ as taxas de fecundidade eram iguais. $\mathrm{O}$ diferencial desses resultados em relação aos de Gompertz ${ }^{6}$ é mínimo. Todos os outros resultados obtidos pelo método de Arriaga ${ }^{5}$ são mais baixos que os obtidos pelos demais métodos. Dentre os métodos considerados, esse mostrou um declínio mais expressivo de fecundidade urbana $(34,6 \%)$. Já o declínio mais expressivo de fecundidade rural foi o medido pela técnica de Gompertz $^{6}(22,24 \%)$.

As taxas específicas de fecundidade de Cabo Verde por situação de domicílio estão também apresentadas nas tabelas 1, 2 e 3 . Como se pode verificar, a fecundidade é sistematicamente mais alta nas áreas rurais comparadas às urbanas, tanto em 1990 quanto em 2000. O diferencial relativo cresce com a idade das mulheres, sendo mais baixo no grupo de 15 a 19 anos, com exceção em alguns casos das mulheres em um outro extremo da idade reprodutiva.

As mulheres das áreas rurais experimentaram uma Taxa de Fecundidade Total (TFT) em 1990 e 2000, respectivamente, de 6,1 e 4,8 filhos, pelo método de Brass, ${ }^{4}$ de 5,7 e 4,8 filhos, pelo método de Arriaga $^{5}$ e de 6,0 e 4,7 filhos, pelo de Gompertz. ${ }^{6}$ Experimentaram, em média, $0,9,0,5$ e 1 filho a mais que as residentes nas áreas urbanas, respectivamente, por Brass, ${ }^{4} \mathrm{Ar}$ riaga $^{5}$ e Gompertz, ${ }^{6}$ em 1990. Em 2000, o diferencial aumentou, as mulheres das áreas rurais passaram a experimentar em média 1,1 e 1,4 filho, respectivamente, por Brass, ${ }^{4}$ e Arriaga ${ }^{5}$ a mais que as urbanas. Pelo método de Gompertz, ${ }^{6}$ a diferença manteve-se em um filho.

Todas as taxas declinaram no período, com exceção da referente às mulheres de 15 a 19 anos, que apresentaram aumentos pelos três métodos utilizados. Contudo, as taxas desse grupo etário de 1990 e 2000 não são estatisticamente diferentes.

O declínio das taxas dos restantes grupos de mulheres ocorreu com intensidade progressivamente maior conforme aumentava a idade. As quedas foram, respectivamente, pelos métodos de Brass, ${ }^{4}$ Arriaga ${ }^{5}$ e Gompertz, ${ }^{6}$ de 19,3\%, $15,0 \%$ e $20,8 \%$, no grupo de 20 a 24 anos; de $34,1 \%, 32,2 \%$ e $31,4 \%$, no grupo de 25 a 29 anos; de $37,0 \%, 35,3 \%$ e $36,0 \%$, no grupo de 30 a 34 anos; de $39,6 \%, 38,6 \%$ e $37,9 \%$, no grupo de 34 a 39 anos; e, finalmente, de $31,1 \%, 30,2 \%$ e $37,8 \%$, no grupo de 40 a 44 anos. 
Tabela 1: Fecundidade total e idade média de fecundidade estimada, segundo técnica de Brass. Cabo Verde, 1990 e 2000.

\begin{tabular}{|c|c|c|c|c|c|c|c|c|c|c|c|c|c|c|c|c|}
\hline \multirow{2}{*}{$\frac{\text { Anos }}{\text { Brass* }}$} & \multicolumn{6}{|c|}{1990} & \multicolumn{6}{|c|}{2000} & & \multicolumn{3}{|c|}{ Variação de TEF (\%) } \\
\hline & Urbano & $\begin{array}{l}\text { Urbano } \\
(\%)\end{array}$ & Rural & $\begin{array}{l}\text { Rural } \\
(\%)\end{array}$ & Total & $\begin{array}{l}\text { Total } \\
(\%)\end{array}$ & Urbano & $\begin{array}{l}\text { Urbano } \\
(\%)\end{array}$ & Rural & $\begin{array}{l}\text { Rural } \\
(\%)\end{array}$ & Total & $\begin{array}{l}\text { Total } \\
(\%)\end{array}$ & & Urbano & Rural & Total \\
\hline$\overline{\text { TFT }^{\dagger}}$ & 5,2 & & 6,1 & & 5,8 & & 3,7 & & 4,8 & & 4,1 & & & $-28,85$ & $-21,31$ & $-29,31$ \\
\hline Grupo de idade & TEF* & & TEF & & TEF & & TEF & & TEF & & TEF & & & & & \\
\hline De 15 a 19 & 0,1105 & 10,7 & 0,1036 & 8,5 & 0,1096 & 9,4 & 0,1055 & 14,4 & 0,1244 & 12,9 & 0,1128 & 13,6 & & $\S$ & $\S$ & $\S$ \\
\hline De 20 a 24 & 0,2341 & 22,6 & 0,2375 & 19,5 & 0,2417 & 20,8 & 0,1707 & 23,2 & 0,236 & 24,4 & 0,1951 & 23,6 & & $-27,1$ & $\S$ & $-19,3$ \\
\hline De 25 a 29 & 0,2604 & 25,1 & 0,2624 & 21,6 & 0,2683 & 23,1 & 0,1643 & 22,4 & 0,1999 & 20,7 & 0,1769 & 21,4 & & $-36,9$ & $-23,8$ & $-34,1$ \\
\hline De 30 a 34 & 0,2059 & 19,9 & 0,2598 & 21,4 & 0,2397 & 20,6 & 0,137 & 18,6 & 0,171 & 17,7 & 0,151 & 18,3 & & $-33,5$ & $-34,2$ & $-37,0$ \\
\hline De 35 a 39 & 0,151 & 14,6 & 0,2183 & 17,9 & 0,192 & 16,5 & 0,1005 & 13,7 & 0,1375 & 14,2 & 0,1159 & 14,0 & & $-33,4$ & $-37,0$ & $-39,6$ \\
\hline De 40 a 44 & 0,0594 & 5,7 & 0,106 & 8,7 & 0,0866 & 7,5 & 0,0438 & 6,0 & 0,0785 & 8,1 & 0,0597 & 7,2 & & $\S$ & $-25,9$ & $-31,1$ \\
\hline De 45 a 49 & 0,015 & 1,4 & 0,0286 & 2,4 & 0,0233 & 2,0 & 0,0132 & 1,8 & 0,0183 & 1,9 & 0,0159 & 1,9 & & $\S$ & $\S$ & $\S$ \\
\hline $\begin{array}{l}\text { média } \\
\% \text { abaixo de } 30 \text { ano }\end{array}$ & 28,9 & 58,4 & & $\begin{array}{l}30,3 \\
49,6\end{array}$ & & $\begin{array}{l}29,7 \\
53,4\end{array}$ & 28,5 & 59,9 & & $\begin{array}{c}28,7 \\
58,0\end{array}$ & & $\begin{array}{l}0,4 \\
58,6\end{array}$ & $-1,4$ & $-1,0$ & & \\
\hline$\% \geq 30$ anos & & 41,6 & & 50,4 & & 46,6 & & 40,1 & & 42,0 & & 41,4 & & & & \\
\hline
\end{tabular}

Fonte: Censos demográficos de 1990 e 2000. Instituto Nacional de Estatística. Cabo Verde. África Ocidental

Tabela 2: Fecundidade total e idade média de fecundidade estimada, de acordo com a técnica de Arriaga. Cabo Verde, 1990 e 2000.

\begin{tabular}{|c|c|c|c|c|c|c|c|c|c|c|c|c|c|c|c|c|}
\hline Anos & & & 1990 & & & & & & 2000 & & & & & Variaç & go de TE & \\
\hline$\overline{\text { Arriaga }{ }^{I I}}$ & Urbano & $\begin{array}{l}\text { Urbano } \\
(\%)\end{array}$ & Rural & $\begin{array}{l}\text { Rural } \\
(\%)\end{array}$ & Total & $\begin{array}{l}\text { Total } \\
(\%)\end{array}$ & Urbano & $\begin{array}{l}\text { Urbano } \\
(\%)\end{array}$ & Rural & $\begin{array}{l}\text { Rural } \\
(\%)\end{array}$ & Total & $\begin{array}{l}\text { Total } \\
(\%)\end{array}$ & & Urbano & Rural & Total \\
\hline$\overline{T F T}$ & 5,2 & & 5,7 & & 5,5 & & 3,4 & & 4,8 & & 4,0 & & & $-34,62$ & $-15,79$ & $-27,29$ \\
\hline Grupo de idade & TEF & & TEF & & TEF & & TEF & & TEF & & TEF & & & & & \\
\hline De 15 a 19 & 0,093 & 9,0 & 0,0827 & 7,2 & 0,0876 & 8,0 & 0,083 & 12,2 & 0,1044 & 10,8 & 0,092 & 11,6 & & $\S$ & $\S$ & $\S$ \\
\hline De 20 a 24 & 0,225 & 21,7 & 0,2145 & 18,8 & 0,2193 & 20,0 & 0,1562 & 23,0 & 0,2348 & 24,3 & 0,1864 & 23,4 & & $-30,6$ & 9,5 & $-15,0$ \\
\hline De 25 a 29 & 0,2617 & 25,3 & 0,2443 & 21,4 & 0,2526 & 23,1 & 0,1531 & 22,6 & 0,2027 & 21,0 & 0,172 & 21,6 & & $-41,5$ & $-17,0$ & $-32,2$ \\
\hline De 30 a 34 & 0,2111 & 20,4 & 0,2459 & 21,5 & 0,2296 & 21,0 & 0,1294 & 19,1 & 0,174 & 18,0 & 0,148 & 18,6 & & $-38,7$ & $-29,2$ & $-35,3$ \\
\hline De 35 a 39 & 0,1586 & 15,3 & 0,2116 & 18,5 & 0,1881 & 17,2 & 0,0971 & 14,3 & 0,1413 & 14,6 & 0,1154 & 14,5 & & $-38,8$ & $-33,2$ & $-38,6$ \\
\hline De 40 a 44 & 0,0659 & 6,4 & 0,1098 & 9,6 & 0,0903 & 8,2 & 0,0444 & 6,5 & 0,086 & 8,9 & 0,063 & 7,9 & & $-32,6$ & $-21,7$ & $-30,2$ \\
\hline De 45 a 49 & 0,0195 & 1,9 & 0,034 & 3,0 & 0,028 & 2,6 & 0,0155 & 2,3 & 0,0237 & 2,5 & 0,0195 & 2,4 & & $\S$ & $\S$ & $\S$ \\
\hline $\begin{array}{l}\text { média } \\
\% \text { abaixo de } 30 \text { anos }\end{array}$ & 29,4 & 56,0 & 30,8 & 47,4 & 30,2 & 51,1 & 28,9 & 57,8 & 29,4 & $\begin{array}{l}29,2 \\
56,0\end{array}$ & & $\begin{array}{r}-0,5 \\
56,6\end{array}$ & $-1,4$ & $-1,0$ & & \\
\hline$\% \geq 30$ anos & & 44,0 & & 52,6 & & 48,9 & & 42,2 & & 44,0 & & 43,4 & & & & \\
\hline
\end{tabular}

Fonte: Censos demográficos de 1990 e 2000. Instituto Nacional de Estatística. Cabo Verde. África Ocidenta 
Tabela 3: Fecundidade total e idade média de fecundidade estimada, de acordo com o métodos de Gompertz. Cabo Verde, 1990 e 2000.

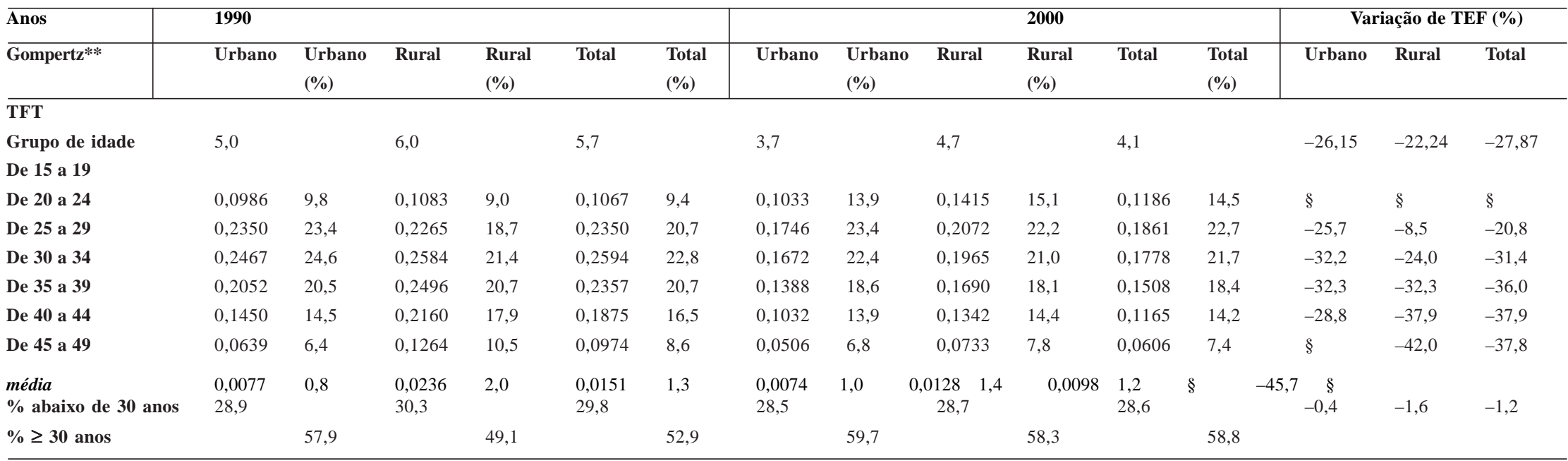

Fonte: Censos demográficos de 1990 e 2000. Instituto Nacional de Estatística. Cabo Verde. África Ocidental

Notas:

* Na estimativa de fecundidade por método de Brass, o nível da fecundidade foi ajustado pela razão $P_{2} / F_{2}$, por se acreditar que para o segundo grupo etário as informações acerca da fecundidade retrospectiva têm precisão mais aceitável. Assume-se que as informações referentes às mulheres jovens não devem conter substancial erro de memória.

$\dagger$ TFT $=$ Taxa de Fecundidade Total.

$\$ \mathrm{TEF}=$ Taxa Específicas de Fecundidade.

$\S$ Taxas de variação entre 1990 e 2000 que não são relevantes

média = Idade média de fecundidade.

If Na estimativa de fecundidade por método de Arriaga, utilizou-se a interpolação linear entre o número médio de filhos por mulher dos censos de 1980 , de 1990 e de 2000.

** Na estimativa de fecundidade por método de Gompertz, há três hipóteses possíveis do comportamento de fecundidade (por exemplo, para o país em 2000, na $1^{\mathrm{a}}$ hipótese se considerou $\alpha=-0,0162, \beta=0,9542$ e TFT $=4,20$; na $2^{\mathrm{a}}$ hipótise $\alpha=-0,0613, \beta=0,8878$ e TFT $=4,38$; e, por último, na $3^{\mathrm{a}}$ hipótese se

considerou $\alpha=-0,0028, \beta=0,9216$ e TFT $=4,13$ ). Decisão: optou-se pela terceira hipótese, por essa parecer apresentar um nível mais próximo da realidade atual. 
Esses dados também apontam para uma ligeira diminuição na idade média da fecundidade para o país como um todo, denotando mudança no padrão por idade da curva dessa variável. O gráfico 1 mostra a mudança de cúspide tardio (de 25 a 29 anos) para precoce (de 20 a 24 anos). Em 1990, a idade média de fecundidade ficou em torno de 30 anos e, em 2000, declinou em um ano, repousando na casa dos 29 anos.

Foi mensurado também a contribuição das variáveis intermediárias nos níveis de fecundidade e nos seus diferenciais. A metodologia utilizada foi do modelo proposto por Bongaarts (s/d):

$$
T F T=C_{m} \times C_{c} \times C_{a} \times C_{i} \times T F
$$

em que $C_{m}, C_{i}, C_{c}$ e $C_{a}$ refletem os fatores inibidores do não-casamento, da amenorréia lactacional, dos anticoncepcionais e do aborto induzido, respectivamente. TFé a taxa de fertilidade. Consideramos um valor fixo de 15,9 para a estimação da taxa de fertilidade.

A taxa de fecundidade cabo-verdiana difere desse valor máximo, principalmente, pela eficácia e o uso de anticoncepcionais, método que se mostrou mais importante no controle de fecundidade. Este apresentou um efeito redutor de $27,2 \%$ ou 4,3 filhos na taxa de fecundidade total.

A amamentação afeta o período de não susceptibilidade pós-parto e é considerada, também, um dos principais fatores inibidores da fecundidade cabo-verdiana, contribuindo para a redução de quase um quarto da taxa de fecundidade total ( $24,1 \%$ ou 3,8 filhos). O não casamento teve o mesmo efeito na inibição da fecundidade que a amamentação. Com contribuição de $23,8 \%$ ou 3,8 filhos para que a fecundidade não atingisse o seu valor máximo.

Gráfico 1 - Taxas específicas de fecundidade estimadas pelos métodos de Brass, Arriaga e Gompertz. Cabo Verde - 1990 e 2000.
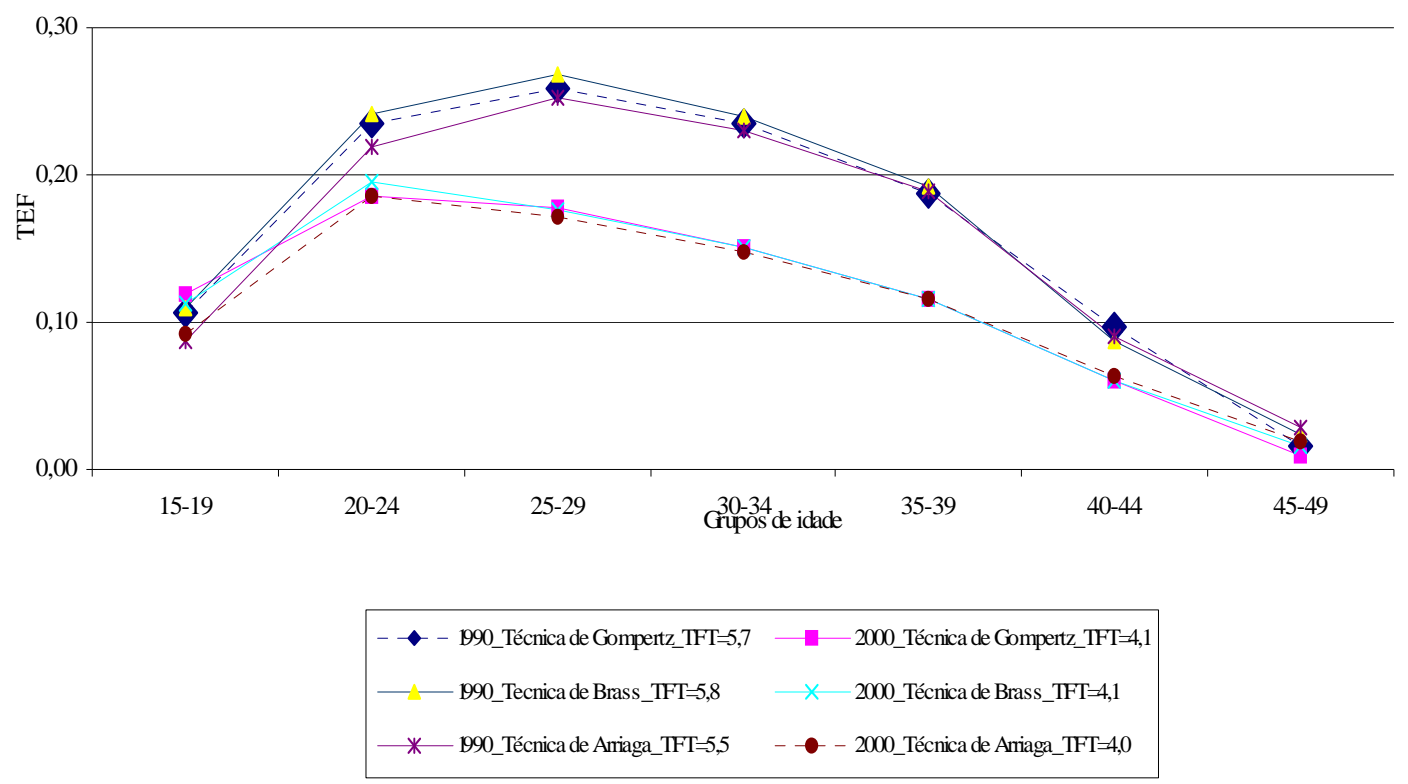

IDSR - Inquérito Demográfico e de Saúde Reprodutiva.

\section{DISCUSSÃO}

Os níveis de fecundidade de um país fazem parte de um elenco de indicadores que orientam na formulação de políticas públicas, em face de redução do volume da população e do seu envelhecimento. Ademais, o aumento da fecundidade pode indicar falta de acesso da população a informações e serviços de saúde reprodutiva. 
Em Cabo Verde, houve um declínio de fecundidade no período de 1990 a 2000. Três métodos foram utilizados para a mensuração de fecundidade. ${ }^{4-6}$ Nesse período, a taxa de fecundidade total declinou em 29,31\% (método de Brass $^{4}$ ), 27,29\% (método de Arriaga ${ }^{5}$ ) e 27,87\% (método de Gompertz ${ }^{6}$ ). Passou de 5,8 filhos para 4,1, de 5,5 filhos para 4,0 e de 5,7 filhos para 4,1, respectivamente pelos três métodos.

O maior declínio relativo de fecundidade nos grupos mais velhos é um fenômeno esperado, dada à experiência dos países que já experimentaram a transição demográfica. ${ }^{9}$

Pirotta, ${ }^{10} \mathrm{em}$ concordância com o resultado desse estudo, mostrou que a única faixa etária em que se observou um aumento na taxa de fecundidade é a das adolescentes. Em todas as outras faixas etárias, que sucedem à adolescência, a taxa de fecundidade apresentou um declínio sistemático. De acordo com a autora, as pesquisas indicam ainda que esteja ocorrendo uma concentração da fecundidade em um intervalo menor do período reprodutivo, com o aumento da presença das mulheres mais jovens na fecundidade total, ou seja, está se assistindo a um rejuvenescimento da fecundidade, bem como Camarano ${ }^{9}$ e Wong e Perpétuo ${ }^{11}$ concluíram.

Segundo Wong e Perpétuo, ${ }^{11}$ o atual perfil de mudança para um padrão reprodutivo essencialmente jovem estaria relacionado a vários fatores, interessando mencionar o timing do início desse processo e o mix da prevalência contraceptiva.

Os dados da tabela 1 e o gráfico 1 são convergentes com os estudos similares, ${ }^{10,11}$ pois parecem confirmar um início mais cedo de fecundidade em 2000 em relação a 1990, independentemente do método utilizado. Isso pode estar associado a uma alta proporção de fecundidade entre as mulheres menores de 20 anos, principalmente nas áreas rurais e no país como um todo, apesar de as taxas desse grupo de mulheres não serem estatisticamente diferentes.

A idade média de fecundidade por situação de domicílio calculada pelos três métodos ${ }^{4-}$ ${ }^{6}$ para 2000 foi em torno de 29 anos, tanto para as áreas urbanas quanto para as rurais. No entanto, em 1990, esse indicador para a área ru- ral foi de 1,4 ano superior ao das áreas urbanas. Isso, em parte, deveu-se à variação positiva e exclusiva das taxas nas primeiras idades (de 15 a 24 anos) na área rural, o que reduziu a sua média em 1,5 ano. A referida média para as mulheres na área urbana diminuiu em apenas cerca de 0,5 ano no período, provavelmente devido à variação nas taxas específicas ter sido mais homogênea.

As mulheres em idade fértil, residentes em áreas urbanas cabo-verdianas, experimentaram em 1990 uma TFT em torno de 5 filhos. Em 2000, esse valor decresceu para aproximadamente 3,5 filhos.

Segundo as três técnicas de estimação, as taxas de fecundidade das mulheres rurais foram de 5,7 e 6,1 filhos em 1990. Embora essas mulheres tenham reduzido a sua fecundidade em 2000, (4,8 filhos segundo técnicas de Arriaga ${ }^{5} \mathrm{e}$ Brass $^{4}$ e 4,7 filhos pela técnica de Gompertz ${ }^{6}$ ), a variação foi menos significativa $(-21,31 \%$, $15,79 \%$ e $-22,24 \%$, respectivamente, segundo as técnicas de Brass, ${ }^{4}$ Arriaga $^{5}$ e Gompertz ${ }^{6}$ que a observada para as mulheres residentes na região urbana. As mulheres urbanas, pelo método de Arriaga, ${ }^{5}$ experimentaram uma variação percentual da TFT, também negativa, de quase o dobro $(-34,62 \%)$ das rurais $(-15,79 \%)$. Nota-se um declínio maior na fecundidade das mulheres urbanas do que das rurais em todas as estimativas obtidas. Isso é um padrão esperado. ${ }^{12,13}$

Em Cabo Verde, à semelhança do que já aconteceu em outros países, tal qual o Brasil, os dados de 2000 mostram que a fecundidade das mulheres rurais de Cabo Verde também está declinando. A implicação disso é que, em 2000, principalmente pelo método de Arriaga, ${ }^{5}$ não houve diferença entre a idade média de fecundidade das mulheres por situação de domicílio.

Destacam-se os diferenciais por situação de domicílio tanto no que se refere à TFT quanto às idades médias da fecundidade calculadas para áreas urbana e rural. Em 1990, os diferenciais na TFT das mulheres entre os quadros domiciliares, urbano e rural foram de $17,31 \%, 9,62 \%$ e $20,0 \%$, respectivamente. Esses diferenciais elevaram-se em 2000, atingindo a casa dos 29,73\%, $41,18 \%$ e $27,0 \%$, respectivamente pelos méto- 
dos de Brass, ${ }^{4}$ Arriaga $^{5}$ e Gompertz. ${ }^{6}$

Da mesma forma, os diferenciais entre as idades médias de fecundidade domiciliares, urbano e rural, em 1990, foram de 1,6 ano, pelos métodos de Brass ${ }^{4}$ e Gompertz; ${ }^{6}$ e de 1,4 ano, pelo de Arriaga. ${ }^{5}$ Esses diferenciais reduziramse de acordo com métodos de Brass ${ }^{4}$ e Gompertz ${ }^{6} \mathrm{em} 2000$. Pelo método de Gompertz, ${ }^{6}$ nesse ano (2000), não se observou diferenças expressivas em idade média de fecundidade por situação de domicílio. Em 2000, para o país como um todo, ela ficou em torno de 29 anos, de acordo com os três métodos. Isso pode ter sido consequiência da queda de fecundidade total, quase em todos os grupos de idade das mulheres, em ambos os domiciliares, com maior intensidade nos grupos de mulheres acima de 25 anos de idade.

A diminuição na idade média de fecundidade das mulheres cabo-verdianas observada para o país como um todo foi extensiva à situação de domicílio, o que poderá ser explicado pela mudança de padrão de fecundidade. $\mathrm{O}$ tipo de mudança também apresentado por cada situação de domicílio foi de deslocamento da cúspide das curvas de fecundidade que, até pelo menos em 1990, concentravam-se no grupo de 25 a 29 anos, ou seja, apresentavam um padrão tardio para o de 20 a 24 anos, sendo padrão caracteristicamente precoce em 2000. Os resultados encontrados ilustram o rejuvenescimento da fecundidade ao longo desses períodos, assim como sua concentração nas primeiras idades das mulheres em qualquer uma das situações de domicílio, embora com maior intensidade nas áreas rurais.

O rejuvenescimento da fecundidade do país como um todo é explicado, principalmente, pelo comportamento das áreas rurais. A tendência ao "rejuvenescimento" no total da fecundidade, considerando o aumento de contribuição de fecundidade das mulheres mais jovens (de 20 a 24), aumentou no período. Passou de 20,8\%, 20\% e 20,7\% do total, em 1990, para 23,6\%, 23,4\% e 22,7\% do total, em 2000, respectivamente pelos métodos de Brass, ${ }^{4}$ Arriaga ${ }^{5}$ e Gompertz. ${ }^{6}$ Já a contribuição das mulheres de grupo de 25 a 29 anos no total de fecundidade diminuiu de $23,1 \%, 23,1 \%$ e $22,8 \%$, em 1990, para 21,4\%, 21,6\% e 21,7\% do total, em 2000, pelos métodos de Brass, ${ }^{4}$ Arriaga ${ }^{5}$ e Gom- pertz ${ }^{6}$ respectivamente.

Oliveira, ${ }^{14}$ no seu estudo sobre a evolução dos níveis e padrões da fecundidade no Brasil, associa esse tipo de "rejuvenescimento" do padrão da fecundidade à redução no número médio de filhos por mulher e concluiu que o país experimentou também uma mudança no padrão de fecundidade. Entre 1990 e 2000, observou-se um rejuvenescimento da idade de maternidade cabo-verdiana, provocado também pelo aumento da participação de fecundidade dos grupos de mulheres de 15 a 19 anos. Essa mudança reflete dois movimentos simultâneos, a redução nos níveis de fecundidade dos grupos de mulheres em idades superiores aos 25 ou 30 anos, associadas, em parte, ao controle da fecundidade quando atingiu o número de filhos desejados e o aumento da fecundidade no grupo de mulheres mais jovens, inclusive entre crianças e adolescentes. Esse último fato requer uma atenção maior das autoridades, devido aos problemas sociais e de saúde que podem ser provocados.

O padrão geral de mudança na estrutura da fecundidade, tanto da população do país como um todo quanto das áreas rurais e urbanas, foi marcado pelo aumento da participação relativa das mulheres jovens até 30 anos de idade e uma conseqüente diminuição da participação relativa das mulheres acima de 30 anos. Esse padrão geral de mudança é característico das populações que experimentaram declínio expressivo de fecundidade. Deve-se, no entanto, observar que o aumento de fecundidade do grupo de 15 a 19 anos, segundo Fernandez e Carvalho, ${ }^{15}$ foge aos padrões da teoria da transição demográfica. Na verdade, enquanto entre 1990 e 2000 as taxas de fecundidade dos grupos de idade acima de 20 anos declinaram, exceto para a área rural quando medida pela técnica de Arriaga ${ }^{5}$, a fecundidade do grupo de 15 a 19 anos experimentou um aumento entre as mulheres de população total, devido ao aumento observado na população rural.

Também, de maneira convergente, observou-se que houve um aumento da fecundidade entre as adolescentes cabo-verdianas. Infere-se que esse fato tenha ocorrido por situações culturais, tais como a liberação sexual, o processo crescente de urbanização, transformações soci- 
ais, prolongamento do período de formação educacional, menor prevalência de métodos anticonceptivos e processos biológicos, tal qual a menarca antecipada, que isoladamente contribui para o aumento do período fértil da mulher desde idades precoces.

As razões da fecundidade cabo-verdiana não atingir a sua taxa máxima de 15,9 filhos. Ou seja, supondo uma taxa que seria observada caso todas as mulheres se casassem, se nenhuma usasse métodos anticoncepcionais e tampouco amamentassem. A taxa de fecundidade cabo-verdiana difere desse valor máximo, principalmente, pela eficácia e o uso de anticoncepcionais, método que se mostrou mais importante no controle de fecundidade. Este apresentou um efeito redutor de $27,2 \%$ ou 4,3 filhos na taxa de fecundidade total.

A amamentação afeta o período de não susceptibilidade pós-parto e é considerada, também, um dos principais fatores inibidores da fecundidade cabo-verdiana, contribuindo para a redução de quase um quarto da taxa de fecundidade total ( $24,1 \%$ ou 3,8 filhos). O não-casamento teve o mesmo efeito na inibição da fecundidade que a amamentação. Contribuiu em $23,8 \%$ ou 3,8 filhos para que a fecundidade não atingisse o seu valor máximo. Esperava-se que o não-casamento tivesse uma importância maior, como acontecem nos outros países. Por exemplo, no caso do Nordeste do Brasil, o não-casamento contribuiu em $44 \%$ para as mulheres que cresceram nas capitais e $20 \%$ para as mulheres que cresceram nas áreas rurais, segundo Camarano ${ }^{16}$.

\section{CONCLUSÃO}

Cabo Verde obteve um decréscimo na fecundidade das mulheres com 20 anos ou mais e aumento da fecundidade naquelas com menos de 20 anos. $\mathrm{O}$ uso de contraceptivos foi o determinante de maior relevância no processo de declínio de fecundidade das mulheres de Cabo Verde.

\section{Agradecimentos}

Os autores agradecem ao apoio financeiro do Instituto Brasileiro de Geografia e Estatística (IBGE). Agradecem também ao Vitor Engrácia Valenti pela contribuição na revisão e formatação do manuscrito.

\begin{abstract}
A country's fertility levels are part of a list of indicators that guide the formulation of public policies, in view of population reduction and population aging. An increase in fertility may indicate the population's lack of access to reproductive health information and services. Therefore, our aim is to analyze Cape Verdean women's fertility and the contribution of proximal determinants of fertility. The analysis and measurement of the behavior of the women's fertility were performed through indirect methods of fertility estimation based on census data. The analysis of the parameters near fertility was based on DHS (1998). The data showed a reduction of 1.7, 1.5 and 1.6 children per woman at the end of the reproductive period according to the three methods (Brass, Arriaga and Gompertz) between 1990 and 2000. Rates were higher for women who lived in rural areas. Fertility has been decreasing in the country and the use of contraceptives was the most relevant parameter regarding the fertility decline process of women from Cape Verde, Africa.
\end{abstract}

Keywords: Fertility rate; contraception; Western Africa.

\section{REFERÊNCIAS}

1. Montoya JM, Bernal A, Borrero C. Diagnostics in assisted human reproduction. Reprod Biomed Online. 2002;5(2):198-210.

2. Camlin CS, Garenne M, Moultrie TA. Fertility trend and pattern in a rural area of South Africa in the context of HIV/AIDS. Afr J Reprod Health. 2004;8(2):38-54.
3. Day LH. Recent fertility trends in industrialized countries: toward a fluctuating or a stable pattern? Eur J Popul. 1995;11(3):275-88.

4. Brass W. The demography of Tropical Africa. Princeton University Press; 1968.

5. Arriaga EE. Estimating fertility from data on children ever born age of mother. US Bureau of the Census; 1970. 
6. Newell C. Methods and models in demography. London: Behaven Press; 1989.

7. Albuquerque FRPC, Oliveira JC, Pereira NOM. Estimativas de fecundidade para o Brasil com base nas técnicas de Brass e de Arriaga. Rev Bras Estat Pop. 1985;46(8):181-2.

8. Goldberg HI, Rodrigues W, Arruda JM, Morris L. Uso de pesquisas de saúde materno infantil e planejamento familiar para análise de determinantes próximos da fecundidade. Rev Bras Est Pop. 1984;2:945-59.

9. Camarano AA. Fertility transition in Brazil in the twentieth: a comparative study of three areas [tese]. London: The London School of Economics and Political Science, University of London; 1996.

10. Pirota KCM. Juventude e saúde reprodutiva: valores e condutas relacionados com a contracepção entre universitários [resumo]. In: Encontro nacional de estudos populacionais; 2002 Nov 13; Caxambu, Brasil. Caxambu: ABEP; 2002. p. 20.

11. Wong LR, Perpétuo IHO. A fecundidade das Minas Gerais nos anos 90: estabilidade e convergência no nível de reposição [texto completo]. In:
Seminário sobre a economia mineira; 2000 Out 15; Diamantina, Brasil. Minas Gerais: 2000. p. 721-742.

12. Canazas VMA. A fecundidade no Peru: evidências tendências e indícios de convergência [texto completo]. In: Encontro nacional de estudos populacionais; 1996 Set 21; Caxambu, Brasil. Encontro nacional de estudos populacionais. Caxambu: ABEP; 1996. p. 1325-90.

13. Gupta N, Leite IC. Tendências e determinantes da fecundidade entre adolescentes no Nordeste do Brasil. Persp Int Plan Fam. 2001;45:24-9.

14. Oliveira ATR. Notas sobre a evolução dos níveis e padrão da fecundidade no Brasil [acesso em 2004 Set 6]. Disponível em: http://www.univercidade.edu.

15. Fernandez RE, Carvalho JAM. Evolução da fecundidade no Brasil, período de 1957-1979: aplicação da técnica dos filhos próprios para se estimar a fecundidade ano a ano. Rev Bra Est Pop. 1986;2:67-86.

16. Camarano AA. A queda da fecundidade no Nordeste: uma aproximação aos determinantes. In: BEMFAM. Fecundidade, anticoncepção e mortalidade infantil. Rio de Janeiro: BEMFAM, 1994. 